\title{
Comparación interlaboratorial de cronómetros 2014
}

\section{Stopwatch Interlaborator y Comparison}

\section{Oscar Fallas-Cordero 1 , Isabel Castro-Blanco ${ }^{2}$, Harold Sánchez-Vargas ${ }^{3}$}

Fecha de recepción: 2 de diciembre del 2014

Fecha de aprobación: 28 de marzo del 2015

Fallas-Cordero, O; Castro-Blanco, I; Sánchez-Vargas, H.

Comparación interlaboratorial de cronómetros 2014. Tecnolo-

gía en Marcha. Vol. 28, №3, Julio-Setiembre. Pág 64-73.

1 Laboratorio Metrológico de Variables Eléctricas. Instituto Costarricense de Electricidad, Apartado 485-2050. San Pedro, Costa Rica. Correo electrónico: ofallasc@ice.go.cr

2 Laboratorio Metrológico de Variables Eléctricas. Instituto Costarricense de Electricidad, Apartado 485-2050. San Pedro, Costa Rica. Correo electrónico: bcastro@ice.go.cr)

3 Laboratorio Metrológico de Variables Eléctricas. Instituto Costarricense de Electricidad, Apartado 485-2050. San Pedro, Costa Rica. Correo electrónico: hsanchez@ice.go.cr 


\title{
Palabras clave
}

Cronómetros; comparación interlaboratorial; patrón viajero; calibración.

\section{Resumen}

Los cronómetros y temporizadores se utilizan para numerosas aplicaciones y se encuentran entre los dispositivos más comunes calibrados por laboratorios de metrología. Las calibraciones de cronómetros suelen realizarlas tanto los laboratorios secundarios, como los laboratorios estatales o privados. Sin embargo, en países pequeños, el instituto nacional de metrología (INM) a menudo acepta cronómetros para la calibración con el patrón nacional.

En este trabajo se describe y presentan los resultados de una comparación de cronómetros que se llevó a cabo en Costa Rica, entre marzo del 2014 hasta octubre del mismo año, por el Laboratorio Metrológico de Variables Eléctricas (LMVE) del Instituto Costarricense de Electricidad (ICE), como INM designado en Costa Rica para Electricidad, Magnetismo, Tiempo y Frecuencia. La comparación involucró 6 laboratorios participantes y un cronómetro patrón viajero.

\section{Keywords}

Stopwatches; interlaboratory comparison; travel standard; calibration.

\begin{abstract}
Stopwatches and timers are used for numerous applications and are among the most common devices calibrated metrology laboratories. Timer's calibrations are typically carry out by secondary laboratories, as well as state or private laboratories. However, in small countries, the national metrology institute (NMI) often accepts timers for calibration with the national standard.

This paper presents and describes the results of a timer comparison conducted in Costa Rica, from March 2014 until October 2014, by the Instituto Costarricense de Electricidad (ICE)Laboratorio Metrológico de Variables Eléctricas (LMVE) as a designated NMI in Costa Rica for Electricity, Magnetism, Time and Frequency. The comparison involved six participating laboratories and a standard stopwatch traveler.
\end{abstract}

\section{Introducción}

La comparación de cronómetros entre laboratorios, se realiza con el propósito de comparar las capacidades de medición de los laboratorios que ofrecen calibraciones de cronómetros a sus clientes o las utilizan en sus procesos, para mejorar sus métodos de calibración, además de ampliar la colaboración metrológica que ha existido recientemente entre laboratorios que realizan calibraciones en variables de tiempo y frecuencia y el LMVE.

El nivel de experiencia entre los laboratorios varió ampliamente. Algunos de los laboratorios realizan calibraciones rutinariamente mientras que otros no tenían experiencia previa y calibraron un cronómetro por primera vez. Por esta razón, a cada participante se le permitió seleccionar su propio método de calibración, basados en la instrumentación y la experiencia disponibles. La única restricción correspondió a que los laboratorios no podían abrir el cronómetro bajo ninguna 
circunstancia. Cada participante también fue responsable de realizar su propia estimación de la incertidumbre de medición, utilizando métodos coincidentes con los descritos en la GUM ${ }^{4}$.

El Laboratorio Metrológico de Variables Eléctricas (LMVE) fue el laboratorio piloto para la comparación.

El cuadro 1 muestra los laboratorios participantes y los contactos técnicos.

Cuadro 1. Laboratorios participantes y contactos ofrecidos.

\begin{tabular}{|c|c|c|}
\hline Laboratorio & Persona de contacto & Teléfono \\
\hline $\begin{array}{c}\text { SCM Metrología y } \\
\text { Laboratorios S.A }\end{array}$ & Helber Meneses Navarro & 24315252 \\
\hline Elvatron & Oscar Sibaja Seravalli & 22429960 ext. 315 \\
\hline MET-CAL & $\begin{array}{l}\text { Rolando Molina S. } \\
\text { Karen Picado }\end{array}$ & 22864286 \\
\hline CAMERICA & Luis Abarca Camacho & 22802886 \\
\hline $\begin{array}{c}\text { Laboratorio Químico de } \\
\text { Aceites y Combustibles }\end{array}$ & Ana Lucrecia Montero Aguilar & 25011081 \\
\hline RECOPE & Patricia Soto & 25503500 ext. 3858 \\
\end{tabular}

\section{Programación y logística de la comparación}

Para la circulación del cronómetro patrón se propuso un circuito de traslado adecuado entre los laboratorios participantes y el laboratorio piloto, que consistió en la mediciones intermedias por el LMVE tras la medición de dos participantes, lo anterior con el fin de obtener una verificación del valor del patrón de manera periódica tras la movilización a lo largo de la ronda de la comparación.

Estuvo considerado como parte del tiempo de medición, el tiempo requerido para la estabilización y mediciones en cada laboratorio, así como el tiempo para el trasporte adecuado. Los participantes cumplieron con la agenda pactada por lo que los tiempos estimados se consideran adecuados para el ejercicio en cuestión.

El cuadro 2 muestra la programación de mediciones, en donde se puede ver que el LMVE efectuó sus mediciones también al inicio y al final de la comparación. 
Cuadro 2. Programa de mediciones.

\begin{tabular}{|c|c|c|}
\hline Laboratorio & Fecha de medición & Fecha de transporte \\
\hline LMVE & $2013-10-31$ a 2014-03-21 & 2014-03-24 a 2014-03-28 \\
\hline $\begin{array}{c}\text { SCM Metrología y } \\
\text { Laboratorios S.A. }\end{array}$ & 2014-03-31 al 2014-04-18 & 2014-04-21 al 2014-04-25 \\
\hline Elvatron & $2014-04-28$ al 2014-05-09 & 2014-05-12 al 2014-05-16 \\
\hline LMVE & $2014-05-19$ al 2014-05-30 & 2014-06-02 al 2014-06-06 \\
\hline MET-CAL & $2014-06-09$ al 2014-06-20 & 2014-06-23 al 2014-06-27 \\
\hline RECOPE & $2014-06-30$ al 2014-07-11 & 2014-07-14 al 2014-07-18 \\
\hline LMVE & $2014-07-21$ al 2014-08-01 & 2014-08-04 al 2014-08-08 \\
\hline CAMERICA & $2014-08-11$ al 2014-08-22 & 2014-08-25 al 2014-08-29 \\
\hline $\begin{array}{c}\text { Laboratorio Químico de } \\
\text { Aceites y Combustibles }\end{array}$ & $2014-09-01$ al 2014-09-12 & 2014-09-15 al 2014-09-19 \\
\hline LMVE & $2014-09-22$ al 2014-10-03 & N.A \\
\hline
\end{tabular}

El programa se completó en el periodo esperado. Las características técnicas del cronometro patrón viajero se mantuvieron estables durante este período, lo que permite establecer una comparación válida.

\section{Mesurando}

El mesurando se definió como la desviación fraccional de frecuencia del cronómetro (adimensional), que se puede determinar mediante la medición de la frecuencia o el tiempo, debido a la relación:

Donde

$$
\frac{\Delta f}{f}=-\frac{\Delta t}{T}
$$

$$
\frac{\Delta f}{f} \text { es la diferencia entre la frecuencia medida y la frecuencia nominal, divida dicha diferencia }
$$

por la frecuencia nominal y- $\frac{\Delta t}{T}$ es el cambio en el tiempo dividido por el periodo de medición.

\section{Cronómetro patrón viajero}

El patrón viajero que se utilizó corresponde a un cronómetro marca Citizen (figura 1), propiedad del ICE, código I-3659, con una resolución de 0,01 s, pantalla digital, botones de inicio y parada. EI ICE-LMVE mantiene un historial de la estabilidad del patrón viajero. 
Su base de tiempo es un oscilador de cristal de $32768 \mathrm{~Hz}$, similar al oscilador que se encontrar en un reloj de pulsera de cuarzo.

La pantalla se restablece después de un intervalo de tiempo máximo de 9 horas, 59 minutos, $59,99 \mathrm{~s}$, pero los intervalos más largos se pueden medir si el operador calcula el número transcurrido de ciclos de 10 horas.

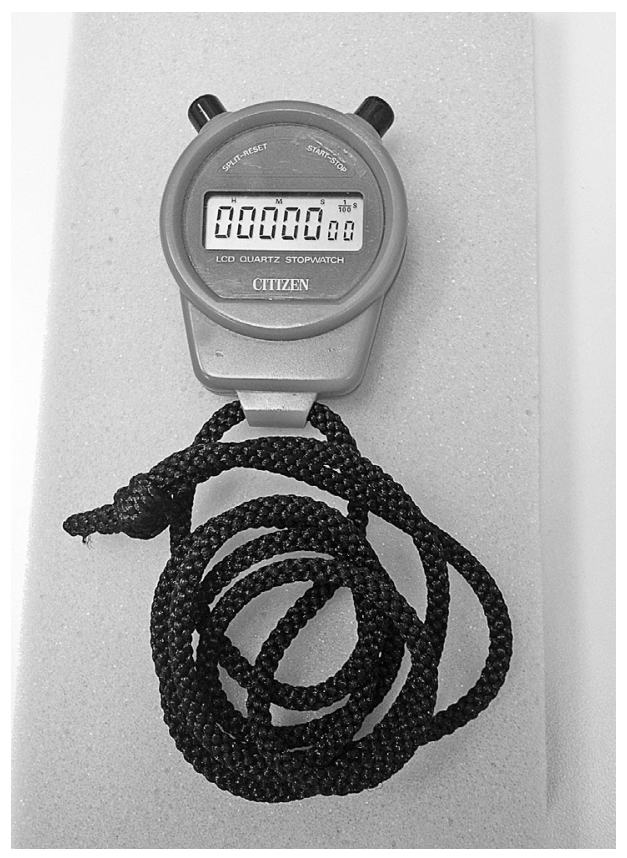

Figura 1. Cronometro utilizado como patrón viajero.

El cronometro viajó en una caja de protección destinada para su traslado. Cada participante usó sus procedimientos normales para el traslado y entrega del equipo.

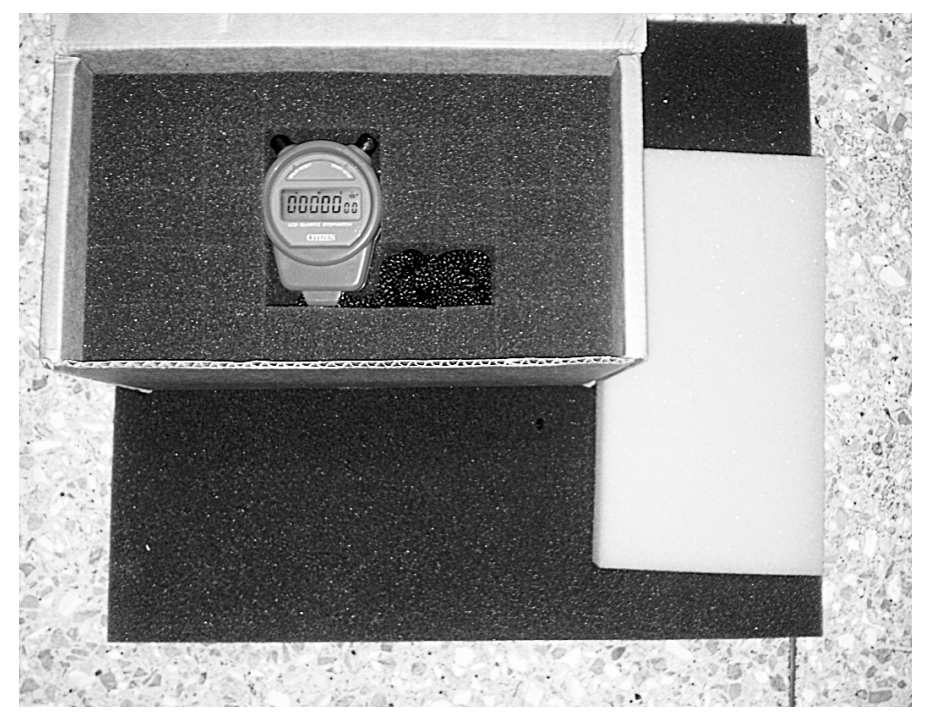

Figura 2. Cronometro en caja utilizada para el transporte 
El LMVE utilizó el método de calibración de base de tiempo mediante la medición por comparación directa de la frecuencia del oscilador de cuarzo, con un contador de frecuencia con su base de frecuencia alimentada desde el oscilador de cesio, el cual es la realización nacional del segundo y es comparado con el Sistema Internacional de Unidades (SI) mediante el Sistema SIM TF (http://tf.nist.gov/sim/simnet.htm).

Las mediciones realizada por el laboratorio piloto corresponden a la diferencia entre la frecuencia medida y la frecuencia nominal del cronometro patrón $(32768 \mathrm{~Hz}$ ). Las mediciones se realizaron cada segundo, de manera consecutiva, durante tres días. Este método de calibración está definido en el alcance de acreditación vigente del LMVE ante el Ente Costarricense de Acreditación (ECA, http://www.eca.or.cr/acr_lab.php?t=c). El valor declarado por el LMVE es de $2.0 \mathrm{E}-10 \mathrm{~Hz} / \mathrm{Hz}(k=2)$, para intervalos de promediación que van desde $1 \mathrm{~s}$ a $86400 \mathrm{~s}$. La mayor contribución a la incertidumbre de la medición por el LMVE corresponde a la variabilidad de las mediciones de la base de tiempo del cronómetro.

El cuadro 3 muestra los resultados de calibración del cronómetro patrón:

Cuadro 3. Resultados de calibración inicial y final del cronómetro patrón.

\begin{tabular}{|c|c|c|c|c|c|}
\hline & Calibración & $\begin{array}{c}\Delta \mathrm{f} / \mathrm{f}(\mathrm{Hz} / \mathrm{Hz}, \\
\left.\text { partes en } 10^{6}\right)\end{array}$ & $\Delta \mathrm{f}(\mathrm{Hz})$ & $s /$ día & $\begin{array}{c}U(k=2, \mathrm{~Hz} / \mathrm{Hz}, \\
\left.\text { partes en } 10^{8}\right)\end{array}$ \\
\hline \multirow{2}{*}{$\begin{array}{c}\text { Cronómetro } \\
\text { Patrón }\end{array}$} & Inicial & 2,52 & 0,083 & 0,22 & 3,75 \\
\cline { 2 - 6 } & Final & 2,37 & 0,078 & 0,21 & 7,47 \\
\hline
\end{tabular}

La desviación fraccional de frecuencia cambió aproximadamente en -1.48 $\times 10^{-7} \mathrm{~Hz} / \mathrm{Hz}$ durante los 178 días transcurridos desde la calibración inicial hasta la calibración final, efectuadas por el LMVE. Se nota que el error acumulado en tiempo (s / día) en el cuadro 3 fue menor a un segundo, lo que es muy bueno comparado con valores típicos de especificaciones de cronómetros (30 s por mes, alrededor de 1 s por día).

\section{Formato de presentación de datos y descripción de los métodos de calibración}

Se pidió a los participantes presentar sus resultados utilizando el formulario que se muestra en el cuadro 4. No todos los participantes siguieron el formato, y alguna información se omitió. Sin embargo, la información recolectada de cada participante fue suficiente para establecer una base para la comparación. 
Cuadro 4. Formulario para entrega de resultados

\begin{tabular}{|c|c|c|}
\hline \multicolumn{3}{|c|}{ Fecha de la calibración } \\
\hline Fecha de inicio & aaaa-mm-ddhh:mm:ss & \\
\hline Fecha de finalización & aaaa-mm-ddhh:mm:ss & \\
\hline \multicolumn{3}{|c|}{ Configuración de la calibración } \\
\hline \multicolumn{3}{|c|}{ Responsable de la toma de datos } \\
\hline \multicolumn{3}{|l|}{ Patrón utilizado } \\
\hline \multicolumn{3}{|l|}{ Trazabilidad } \\
\hline \multicolumn{3}{|l|}{ Método utilizado } \\
\hline \multicolumn{3}{|c|}{ Condiciones Ambientales } \\
\hline \multicolumn{2}{|l|}{ Temperaturamáxima } & \multirow{2}{*}{${ }^{\circ} \mathrm{C}$} \\
\hline \multicolumn{2}{|l|}{ Temperaturamínima } & \\
\hline \multicolumn{2}{|l|}{ Humedadmáxima } & \multirow{2}{*}{$\%$} \\
\hline \multicolumn{2}{|l|}{ Humedadmínima } & \\
\hline \multicolumn{3}{|c|}{ Resultado de la calibración } \\
\hline Item & Valor & Unidad \\
\hline $\begin{array}{c}\text { Desviación Fraccional de } \\
\text { Frecuencia }\end{array}$ & & $\mathrm{s} / \mathrm{s}$ \\
\hline Incertidumbre & & $\mathrm{s} / \mathrm{s}$ \\
\hline Factor de cobertura & & \\
\hline
\end{tabular}

Como se señaló anteriormente, cada laboratorio seleccionó su método de calibración. Sin embargo, se acordó describir el método que eligieron, lo cual se muestra en el cuadro 5. A continuación, se describen brevemente los diferentes métodos que existen para la calibración de cronómetros.

\section{Método de comparación directa}

El método de comparación directa es el más común utilizado para calibrar cronómetros y temporizadores. Se requiere un equipamiento mínimo, pero tiene incertidumbres de medición mayor que otros métodos.

El método de comparación directa requiere una referencia del intervalo de tiempo trazable al SI. Esta referencia es generalmente una señal de tiempo de audio y en algunos casos una visualización de la hora por un patrón trazable al SI. Las señales de tiempo de audio generalmente se obtienen con una radio de onda corta o un teléfono.

\section{Método totalizado}

El método totalizado elimina parcialmente la incertidumbre de medida de tiempo por la reacción humana, pero requiere un generador de señal calibrado y un contador de frecuencia. El contador se pone en modo TOTALIZADO, con puerta manual. Una señal calibrada de un generador sintetizado es conectada a la entrada del contador, y la frecuencia principal del laboratorio se utiliza como la base de tiempo externa para el sintetizador. No se necesita una 
referencia externa para el contador porque el operador realiza el control de tiempo de puerta del contador. La frecuencia debe tener una período, al menos de un orden de magnitud menor que la resolución del cronómetro. Por ejemplo, si el cronómetro tiene una resolución de 0,01 s (10 ms), se utiliza una frecuencia de $1 \mathrm{kHz}$ (período de $1 \mathrm{~ms}$ ). Esto proporciona al contador un dígito más de resolución que el cronómetro.

Para empezar la medición, se inicia el cronómetro y manualmente se abre la puerta del contador al mismo tiempo. Una forma de hacerlo es presionando el botón de arranque y parada del cronómetro contra el botón de inicio del contador. Otro método consiste en presionar el botón de inicio / parada del cronómetro con una mano y presionar simultáneamente el botón inicio / parada del contador con la otra mano. Después de un período adecuado de tiempo (determinado por los requisitos de calibración del cronómetro o el temporizador que se está calibrando) se usa el mismo método para detener el cronómetro y cerrar simultáneamente la puerta del contador.

\section{Método Base Tiempo}

El Método Base Tiempo es el procedimiento de medición preferido para calibraciones de cronómetros y temporizadores, ya que introduce la menor incertidumbre en la medición. Debido a que se mide la base de tiempo del cronómetro directamente, el tiempo de respuesta del operador no es un factor de calibración considerado. El método exacto de medición de la base de tiempo del cronómetro depende del tipo de cronómetro o temporizador que se está calibrando. Si la unidad tiene un cristal de cuarzo como base de tiempo, un sistema inductivo o acústico se puede utilizar para supervisar la frecuencia de $32768 \mathrm{~Hz}$ del oscilador del cronómetro con un contador de frecuencia calibrado (la medición se envía a un amplificador para aumentar la intensidad de la señal).

\section{Resultado de mediciones}

Las condiciones ambientales, los resultados de medición y la incertidumbre estimada se muestran en el cuadro 5.

Cuadro 5. Resultados de los participantes

\begin{tabular}{|c|c|c|c|c|c|c|}
\hline \multirow[b]{2}{*}{ Laboratorio } & \multirow{2}{*}{$\begin{array}{c}\text { Intervalo de } \\
\text { temperatura } \\
\left({ }^{\circ} \mathrm{C}\right)\end{array}$} & \multirow{2}{*}{$\begin{array}{l}\text { Intervalo de } \\
\text { humedad } \\
(\%)\end{array}$} & \multirow[b]{2}{*}{ Método } & \multicolumn{2}{|c|}{ Resultado } & \multirow{2}{*}{$\begin{array}{c}U(k=2) \\
\text { (partes en } \\
\left.10^{6}\right)\end{array}$} \\
\hline & & & & $\begin{array}{c}\mathrm{Hz} / \mathrm{Hz} \\
\text { (partes en } \\
10^{6} \text { ) }\end{array}$ & s/día & \\
\hline LMVE & 18,0 a 23,0 & 30 a 70 & Base de tiempo & 2,49 & 0,21 & 0,042 \\
\hline Lab1 & 17,1 a 23,8 & 47 a 61 & Comparación directa & 2,42 & 0,209 & 0,29 \\
\hline Lab2 & 22,3 a 22,5 & 62 a 68 & Totalizado & $-0,428$ & 0,037 & 4,05 \\
\hline Lab3 & 20 a 22 & 55 a 61 & $\begin{array}{c}\text { Comparación directa } \\
\text { contra patrón }\end{array}$ & 3,27 & 0,283 & 5,8 \\
\hline Lab4 & 22,2 a 23,8 & 48,3 a 63,6 & Comparación directa & 1,3 & 0,112 & 4,3 \\
\hline Lab5 & & & $\begin{array}{c}\text { Comparación directa } \\
\text { contra patrón }\end{array}$ & 2,76 & 0,239 & 0,321 \\
\hline Lab6 & 22,1 a 22,1 & 64 a 70 & $\begin{array}{l}\text { Comparación directa } \\
\text { contra cronometro patrón }\end{array}$ & 2,65 & 0,23 & 0,983 \\
\hline
\end{tabular}


Como se ha indicado anteriormente, cada participante fue responsable de proveer su propia estimación de la incertidumbre de la medición, pero el formulario de presentación (cuadro 4) no exigía a los participantes incluir el análisis de la incertidumbre total; sin embargo, el análisis completo fue proporcionado por cuatro de los laboratorios. Este análisis no se incluye en este informe, sin embargo, la mayoría utiliza una variación del método de suma de la raíz de los cuadrados de cada componente de incertidumbre,

$$
U=k \sqrt{U_{A}^{2}+U_{B}^{2}}
$$

Donde,

$k=$ es el factor de cobertura (2 en todos los casos), es el cuadrado de la incertidumbre evaluada Tipo A y es el cuadrado de la incertidumbre evaluada Tipo B.

Para los laboratorios que mostraron su análisis de la incertidumbre mediante la comparación o totalizan métodos directos, como se esperaba, el tiempo de reacción humana es la mayor fuente de incertidumbre. En el método de base de tiempo, el mayor aporte es la incertidumbre del oscilador de referencia y la incertidumbre contribuida por los sensores e instrumentación. Hay varios ejemplos en los que las estimaciones de la incertidumbre parecen claramente ser sobreestimadas o bien subestimadas, y hay una considerable variación de los resultados de las mediciones reportadas por los diversos laboratorios, como puede verse en la figura 3.

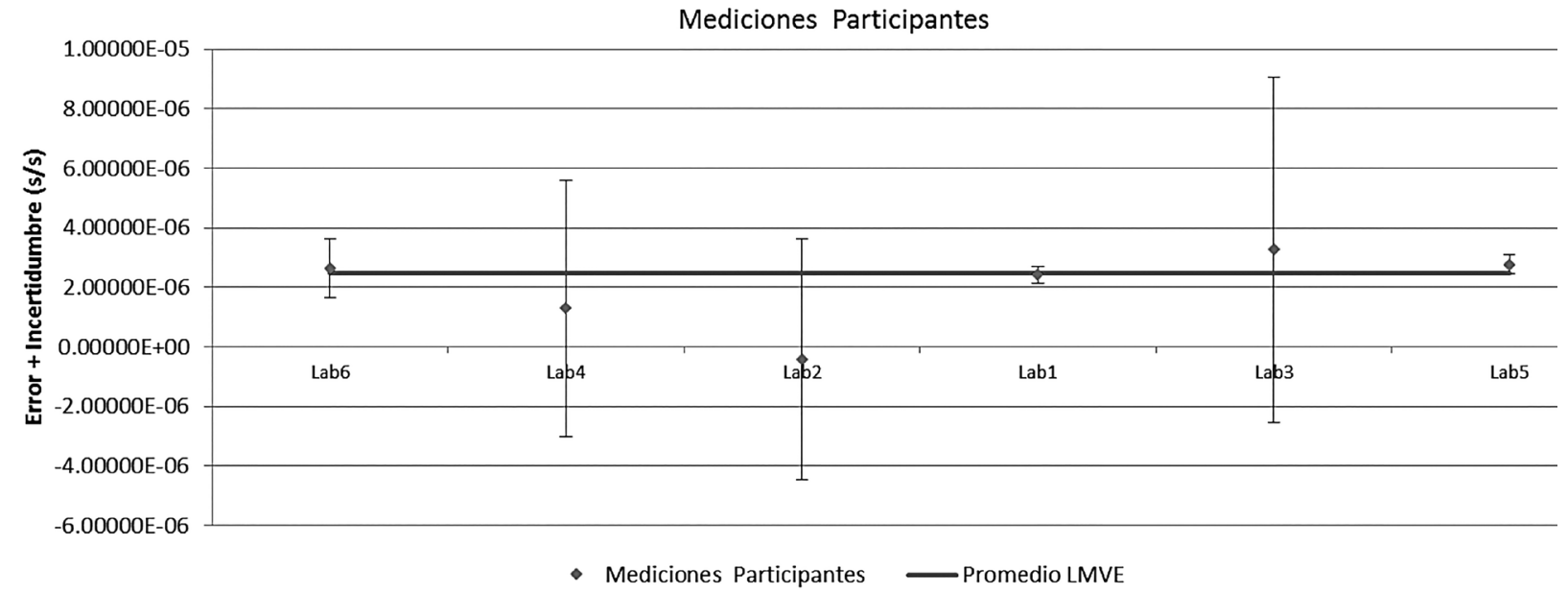

Figura 3.Resultados de los participantes

\section{Resumen y conclusiones}

La comparación mostró diferencias a considerar entre los participantes, por lo que se debe tomar en cuenta que algunos laboratorios no tenían experiencia previa con calibraciones cronómetro y que se utilizaron diferentes métodos de calibración. Los resultados obtenidos en esta comparación muestran que, aunque hay diferencias en los métodos de calibración y en las incertidumbres de los participantes, todos fueron capaces de brindar buenos resultados en esas condiciones. 
Los conocimientos adquiridos durante la comparación podrían ser utilizados para desarrollar un procedimiento estándar para la calibración de cronómetros para los laboratorios involucrados. Aún más importante es que estos procedimientos se podrían distribuir a los laboratorios secundarios e industriales que tienen un gran número de cronómetros para calibrar.

También se espera que, en futuras comparaciones interlaboratorios, se dé un trabajo para mejorar las capacidades de calibración y medición de los participantes, y fortalecer aún más la cooperación entre los laboratorios. Esto podría incluir comparaciones de cronómetros adicionales, o comparaciones de los osciladores de precisión media, como los osciladores de cuarzo horno controlado utilizados en los equipos de prueba, entre otros.

\section{Referencias}

Diaz H. and Postigo H., (September 2013), "Calibración de cronómetros digitales por el método de inducción con una incertidumbre de medición $\leq$ 0,1 $\mathrm{s} / \mathrm{s}$ ", Proceedings of 2013 SEMETRO Conference, Buenos Aires, Argentina, 4 p. Obtenido desde: http://tf.nist.gov//sim/Papers/Diaz_Postigo_SEMETRO_2013.pdf.

Gust J. C., R. Graham M., and Lombardi M. A., (January 2009), "Stopwatch and Timer Calibrations",(2009 edition), NIST Special Publication 960-12, 66 p. Obtenidodesde:http://tf.nist.gov/sim/papers.htm.

Jiménez J. and Sánchez H., (June 2009), "Calibración de Cronómetros Mediante la Medición de la Frecuencia del Oscilador de Cuarzo", Proceedings of 2009 SEMETRO Conference, João Pessoa, Paraíba, Brazil, 3 p. Obtenido desde: http://tf.nist.gov/sim/Papers/Costa_Rica_Stopwatches.pdf.

López-Romero J. M., Lombardi M. A., Díaz-Muñoz N.and de Carlos-Lopez E., (December 2013), "SIM Time Scale", IEEE Transactions on Instrumentation and Measurement, vol. 62, no. 12, pp. 3343-3350. Obtenido desde: http://tf.nist.gov/general/pdf/2613.pdf.

López-Romero J. M., Díaz-Muñoz N., and Lombardi M. A., (August 2009), "Establishment of the SIM Time Scale", INFOSIM: Informative Bulletin of the Interamerican Metrology System - OAS, pp. 39-43. Obtenido desde: http://tf.nist.gov/general/pdf/2431.pdf.

López-Romero J. M., Díaz-Muñoz N., and Lombardi M. A. (October 2008), "Establishment of the SIM Time Scale", Proceedings of the 2008 Simposio de Metrologia, Querétaro, México, 5 pages. Obtenido desde: http:// tf.nist.gov/general/pdf/2306.pdf

Mojica L. M. and Solis R. F., (September 2011), "Sistema de Bajo Costo Para Calibracion Automatizada de Cronometros Digitales", Proceedings of 2011 SEMETRO Conference, Natal, Brazil, 4 p. Obtenido desde:http:// tf.nist.gov/sim/Papers/Mojica_Sistema_de_Bajo_2011.pdf

Mojica L. M. and Solís R. F. (September 2011). "Error de Tiempo en Cronometros Digitales en Base a Mediciones de Intervalo de Tiempo y Frecuencia”, Proceedings of 2011 SEMETRO Conference, Natal, Brazil, 8 p. Obtenido desde: http://tf.nist.gov/sim/Papers/Mojica_Error_de_Tiempo_2011.pdf.

Solís R, Sánchez H, Fallas O, J. Lopez-Romero M, Jiménez F, Postigo H, Perez D, Masi V, Ibrahim A, Lombard M Hoger, B, de Carvalho J R, Orozco G, Reddock T and Trigo L, (February 2011), "AnInterlaboratoryStopwatchComparison in the SIM Region", 7 p. Obtenido desde: http://tf.nist.gov/general/pdf/2571.pdf

Trigo L. and Slomovitz D. (October 2008), "Calibración de cronómetros digitales por método de inducción”, IEEE Encuentro de Energía, Potencia, Instrumentación y Medidas, Montevideo, Uruguay, pp. 21-23. Obtenido desde: http://tf.nist.gov/sim/Papers/Uruguay_Stopwatches.pdf 